\title{
COMPARTIMENTOS DE CARBONO EM LATOSSOLO VERMELHO SOB CULTIVO DE EUCALIPTO E FITOFISIONOMIAS DE CERRADO(1)
}

\author{
Francisco de Alcântara Neto ${ }^{(2)}$, Luiz Fernando Carvalho Leite ${ }^{(3)}$, \\ Emmanuel Arnhold ${ }^{(4)}$, Giovana Alcântara Maciel ${ }^{(3)}$ \& Romero \\ Francisco Vieira Carneiro ${ }^{(2)}$
}

\begin{abstract}
RESUMO
Os objetivos deste trabalho foram avaliar o impacto do cultivo do eucalipto, em diferentes idades, e de fitofisionomias de Cerrado sobre os estoques de C orgânico total e de seus compartimentos e verificar qual dessas frações constituise em indicador mais sensível às mudanças de uso do solo. O estudo foi realizado em Brasília, DF, em Latossolo Vermelho distroférrico sob plantio de eucalipto com três (PEUC-3), seis (PEUC-6) e sete (PEUC-7) anos de idade e sob Cerrado senso stricto, Cerrado denso e campo sujo. Nessas áreas, amostras de solo foram coletadas nas camadas de 0-10 e 10-20 cm, para determinação dos estoques de C orgânico total (COT), carbono da fração leve livre (FLL) e carbono das frações húmicas (ácidos fúlvicos - FAF, ácidos húmicos - FAH e huminas - FH). Em ambas as camadas de solo, observaram-se maiores estoques de COT e de $\mathrm{C}$ nos compartimentos, nas áreas sob Cerrado denso e Cerrado, comparativamente àquelas cultivadas com eucalipto; houve recuperação dos estoques de $\mathrm{C}$ em todos os compartimentos, com o aumento do tempo de cultivo do eucalipto. O C-FLL, seguido do C-FAF e C-FAH, foram considerados os compartimentos mais sensíveis às mudanças no uso do solo, uma vez que as reduções em estoque foram de maior amplitude do que as observadas no COT e C-FH. A análise multivariada, que considera todos os compartimentos conjuntamente, evidenciou maior similaridade entre o Cerrado denso e Cerrado, bem como entre o campo sujo, PEUC-3, PEUC-6 e PEUC7, nas camadas de 0-10 e 10-20 cm.
\end{abstract}

Termos de indexação: matéria orgânica do solo, substâncias húmicas, florestas, uso do solo.

\footnotetext{
(1) Recebido para publicação em abril de 2010 e aprovado em março de 2011.

(2) Professor da Universidade Federal do Piauí - UFPI. Campus Universitário Profa . Cinobelina Elvas, BR 135, Km 03. S/N, Planalto Horizonte, CEP 64900-000 Bom Jesus (PI). E-mail: fneto@ufpi.edu.br; romero@ufpi.edu.br

(3) Pesquisador da Embrapa Meio-Norte. Av Duque de Caxias 5650 Caixa Postal 01, Teresina (PI). Bolsista do CNPq. E-mail: luizf@cpamn.embrapa.br; giovana.maciel@cpac.embrapa.br

(4) Professor da Escola de Veterinária/Departamento de Produção Animal, Universidade Federal de Goiás - UFG. Campus II Samambaia, CEP 74001-970 Goiânia (GO). E-mail: earnhold@pq.cnpq.br
} 


\title{
SUMMARY: CARBON POOLS OF A RED LATOSOL UNDER EUCALYPTUS CULTIVATION AND PHYTOPHYSIOGNOMY OFTHE CERRADO
}

\begin{abstract}
This study aimed to evaluate the impact of the eucalypt cultivation at different ages and of phytophysiognomy of the cerrado biome on total organic $C$ stocks and its pools and to verify which one is more sensitive to land use changes. The study was conducted in Brasilia, Distrito Federal, in a Yellow Latosol under eucalyptus after three (PEUC-3), six (PEUC-6) and seven (PEUC-7) years of cultivation, and under savannah strict sensu, dense savannah and open grassland with scattered shrubs. In these areas, soil samples from the 0-10 and 10-20 cm layers were collected to determine the stocks of total organic carbon (TOC), free light fraction $C$ (FLF) and humic fractions $C$ (fulvic acids - FAF; humic acids - HAF and humin - HF). In both soil layers, higher TOC stocks and C pools were observed in areas under dense savannah and savannah strict sensu than in those under eucalyptus; there was a recovery of soil C stocks with increasing cultivation time of eucalyptus. C-FLF followed by C-FAF and C-HAF were considered most sensitive to land use changes, since the reductions in these stocks were higher than observed for TOC and C-HF. Multivariate analysis, which considers all soil carbon pools simultaneously, showed higher similarity between dense savannah and savannah strict sensu and as well as between open grassland, PEUC-3, PEUC-6 and PEUC-7 in the 0-10 cm and 10-20 cm layers.
\end{abstract}

Index terms: soil organic matter, humic substances, savannah vegetation, land use.

\section{INTRODUÇÃO}

A taxa de conversão da vegetação nativa de Cerrado para culturas anuais, pastagens ou florestas comerciais é de aproximadamente 2,2 $\mathrm{Mha}^{2} \mathrm{ano}^{-1}$ (Bustamante et al., 2006). Essas alterações podem resultar em decréscimo nos estoques de $\mathrm{C}$, em função de incremento no processo erosivo, aceleração da decomposição da matéria orgânica do solo, redução no aporte de material vegetal ou diferenças na qualidade dos resíduos (Lima et al., 2008). Vários fatores alteram a magnitude e a rapidez com que essas mudanças ocorrem, incluindo a natureza do uso, o manejo e tipo de solo, o clima e a vegetação original (Paul et al., 2002).

A substituição da vegetação nativa de Cerrado mais especificamente, por florestas plantadas - tem sido progressivamente praticada (Ferreira et al., 2007), resultando, invariavelmente, em alterações nos estoques de C no solo (Pulrolnik et al., 2009). Contudo, os resultados de diversos estudos, observados, por exemplo, para cultura do eucalipto, têm sido controversos, ou seja, em alguns casos, a substituição da vegetação nativa tem aumentado os estoques de $\mathrm{C}$ orgânico total (COT) (Guo \& Gifford, 2002; Rangel \& Silva, 2007), ao passo que, em outros, esses estoques têm diminuído (Zinn et al., 2005). Essas variações ainda podem ser mais significativas quando se quantificam os compartimentos de $\mathrm{C}$, sobretudo aqueles mais lábeis, com reduzido tempo de residência médio, considerados de maior sensibilidade às ações antrópicas e às mudanças no manejo (Leite et al., 2007; Zagal et al., 2009).

Nesse sentido, a fração leve, considerada compartimento transitório entre a matéria orgânica fresca e a humificada, é considerada de alta labilidade e desempenha importante papel na estrutura e função do solo, especialmente como fonte de energia para organismos heterotróficos e reservatório de C prontamente disponível e de nutrientes para as plantas (Liang et al., 2003; Leite et al., 2007; Laik et al., 2009). Essa fração representa maior percentagem do COT em solos sob vegetação nativa do que sob cultivo, sendo responsiva à umidade do solo, à quantidade de resíduos adicionados e ao grau de perturbação do solo (Haynes et al., 2005).

Moreira \& Malavolta (2004) verificaram que a substituição da vegetação nativa pelo cultivo do eucalipto reduziu os teores de $\mathrm{C}$ da fração leve livre em curto prazo. Adicionalmente, Lima et al. (2008), estudando áreas sob vegetação nativa, pastagem e eucalipto, constataram, além dessa fração, maiores reduções nos estoques de $\mathrm{C}$ das frações ácidos fúlvicos, ácidos húmicos e huminas nas áreas cultivadas, comparativamente à área natural, sendo considerados como indicadores sensíveis da mudança de uso do solo. Assim, este trabalho teve por objetivos avaliar o impacto do cultivo do eucalipto, em diferentes idades, e de fitofisionomias de Cerrado sobre os estoques totais de $\mathrm{C}$ e de seus compartimentos em um Latossolo Vermelho e verificar qual dessas frações constitui-se em indicador mais sensível às mudanças de uso do solo.

\section{MATERIAL E MÉTODOS}

O estudo foi realizado na Fazenda Água Limpa $\left(15^{\circ} 56^{\prime} \mathrm{S}, 47^{\circ} 56^{\prime} \mathrm{W}\right.$ e altitude de $\left.1.100 \mathrm{~m}\right)$, pertencente à Universidade de Brasília, localizada em 
Brasília (DF), em Latossolo Vermelho distroférrico típico textura muito argilosa (Embrapa, 2006). O clima da região, segundo classificação de Köppen, é caracterizado por clima tropical chuvoso (Aw), apresentando temperaturas médias anuais de 18 a $29^{\circ} \mathrm{C}$ e precipitação pluvial média anual em torno de $1.574 \mathrm{~mm}$.

Foram estudadas áreas sob plantio de eucalipto (originalmente sob vegetação nativa de Cerrado e posteriormente sob pastagem com braquiária brizantha) com três (PEUC-3), seis (PEUC-6) e sete (PEUC-7) anos de idade e sob Cerrado senso stricto, Cerrado denso e campo sujo, distanciadas $500 \mathrm{~m}$ uma das outras, e pertencentes à mesma classe de solo $\mathrm{e}$ condições climáticas. O Cerrado senso stricto ocorre geralmente em faixas extensas e contínuas e é caracterizado pela predominância de camada herbácea e por camada lenhosa, que varia de 3 a $5 \mathrm{~m}$ de altura, com cobertura arbórea de 10 a 60 \%, densidade entre 600 a 1.200 plantas lenhosas por hectare, com diâmetro a partir de $5 \mathrm{~cm}$ do Cerrado ralo até o Cerrado denso (Felfili et al., 2004). O Cerrado denso apresenta dossel de 7 a $15 \mathrm{~m}$ de altura, podendo chegar até $20 \mathrm{~m}$ para algumas árvores, com cobertura de 70 a $100 \%$ da área. Além dessas vegetações, têm-se os campos, que são fitofisionomias com predomínio de ervas graminoides e pequenos arbustos. São caracterizados por diversas tipologias, podendo ser denominados campos limpos, quando as árvores são praticamente ausentes, e campos sujos, quando a cobertura arbóreaarbustiva se aproxima de $10 \%$ da área.

Os povoamentos de eucalipto (Eucalyptus grandis) foram estabelecidos em parcelas de 3 x $10 \mathrm{~m}\left(30 \mathrm{~m}^{2}\right)$, em espaçamento de $3 \times 2 \mathrm{~m}$, totalizando 1.667 plantas ha ${ }^{-1}$. No plantio, foram aplicados, na cova, $200 \mathrm{~g}$ de calcário dolomítico e $300 \mathrm{~g}$ da formulação NPK 4-14-8. Na entrelinha do eucalipto com diferentes idades e no Cerrado senso stricto, Cerrado denso e campo sujo, foram abertas três trincheiras para coleta de três amostras simples, para formar uma composta; indeformadas, para determinação da densidade do solo (Embrapa, 1997); e deformadas, para as demais análises, nas camadas de $0-10$ e $10-20 \mathrm{~cm}$. As amostras foram coletadas na época chuvosa, secas ao ar, destorroadas, passadas em peneira de malha de $2,0 \mathrm{~mm}$ e homogeneizadas, para obtenção de terra fina seca ao ar (TFSA). Dessas amostras, retiraram-se subamostras para determinação do C orgânico total (COT), quantificado pelo método de oxidação via úmida, com aquecimento externo (Yeomans \& Bremner, 1988).

A fração leve livre (FLL) foi obtida seguindo-se as recomendações descritas em Sohi et al. (2001) e Machado (2002). A separação da FLL foi feita com base em três repetições analíticas por amostra de solo coletada no campo. Em frascos de centrífuga de $50 \mathrm{~mL}$, foram adicionados $5 \mathrm{~g}$ de TFSA e $35 \mathrm{~mL}$ da solução de iodeto de sódio (NaI), com densidade de 1,8 $\pm 0,1 \mathrm{~kg} \mathrm{~L}^{-1}$. Os frascos com a mistura foram agitados manualmente por $30 \mathrm{~s}$, visando dispersar os agregados instáveis e permitir a flotação da FLL na solução de $\mathrm{NaI}$, e, em seguida, centrifugados a $8.000 \mathrm{rpm}$ por 30 min. Após a centrifugação, a FLL presente na superfície da solução de $\mathrm{NaI}$ foi cuidadosamente aspirada e, em sequência, lavada com água destilada, para eliminação do $\mathrm{NaI}$; em seguida, foi seca em estufa de circulação forçada de ar a $65{ }^{\circ} \mathrm{C}$, por $48 \mathrm{~h}$, pesada, macerada em almofariz de ágata, para posterior determinação do teor de C. As substâncias húmicas (SH) foram quantificadas segundo o método sugerido pela International Humic Substances Society (Swift, 1996), obtendo-se as frações ácidos fúlvicos (FAF), ácidos húmicos (FAH) e huminas (FH), com base na solubilidade dessas frações em soluções ácidas ou alcalinas. O C nas frações FLL, FAF, FAH e FH foi determinado pelo método de oxidação via úmida com aquecimento externo (Yeomans \& Bremner, 1988).

Os estoques totais de $\mathrm{C}$ e dos diferentes compartimentos orgânicos, nas camadas de 0-10 e 10-20 cm, foram calculados multiplicando-se os teores de $\mathrm{C}$ pela massa de solo do Cerrado senso stricto, com o intuito de evitar a interferência da compactação do solo nos estoques de $\mathrm{C}$ do solo, principalmente sob os povoamentos de eucalipto. Os resultados obtidos, em cada profundidade de solo, foram submetidos à análise de variância, seguida pelo teste de Scott-Knott a $5 \%$. Essas análises foram realizadas por meio do software estatístico Assistat (Silva \& Azevedo, 2006). Também foram utilizadas técnicas multivariadas, por meio da construção de um gráfico de componentes principais, da obtenção da medida euclidiana padronizada e do agrupamento pelo método de Tocher, utilizando-se a distância euclidiana padronizada. As análises multivariadas foram realizadas por meio do software R (R. Development Core Team, 2008).

\section{RESULTADOS E DISCUSSÃO}

O estoque de COT foi maior $(p<0,05)$ no solo sob Cerrado denso e Cerrado do que no solo sob os povoamentos de eucalipto, nas três idades avaliadas, nas camadas de $0-10$ e $10-20 \mathrm{~cm}$ (Quadro 1). As reduções em relação ao Cerrado denso variaram de $35,3 \%$ (PEUC-7) a $46,7 \%$ (PEUC-3) e de $27,1 \%$ (PEUC-7) a 30,3 \% (PEUC-3), nas camadas de 0-10 e 10-20 cm, respectivamente. Maiores valores nessas áreas nativas estão relacionados à maior diversidade de plantas e densidade de espécies arbóreas, o que resulta em maior aporte de resíduos vegetais ao solo, especialmente o Cerrado denso, como reportado por Felfili et al. (2004). Por outro lado, diferentemente do Cerrado denso e do Cerrado, o estoque de COT no campo sujo, na camada de $0-10 \mathrm{~cm}$, não diferiu daquele no solo sob PEUC-7 e foi menor do que no solo sob todos os povoamentos de eucalipto, na camada de 10-20 cm (Quadro 1). Esses resultados podem estar associados à maior deposição pelo eucalipto de resíduos 
no solo, na idade de sete anos, e ao maior aporte de $\mathrm{C}$ pelas raízes da cultura, que se distribuem pelo perfil, em diferentes profundidades (Bouillet et al., 2002), diferentemente do campo sujo, que apresenta sistema radicular menos profundo, explorando camadas mais superficiais do solo (Faria et al., 2008).

Apesar de os maiores estoques de COT terem sido observados nas áreas sob florestas nativas (com exceção do campo sujo), comparativamente àquelas sob cultivo do eucalipto, conforme também reportado por outros autores (Zinn et al., 2005), houve recuperação nesses estoques com aumento do tempo de implantação da cultura (PEUC-7 > PEUC-6 > PEUC-3), evidenciado na camada de $0-20 \mathrm{~cm}$, que retrata 0 acúmulo de COT nas duas camadas estudadas (Quadro 1). Isso indica que, em médio e longo prazos, o eucalipto pode aumentar os estoques de COT no solo. Ademais, esses resultados devem ser relativizados, já que se limitam à camada de $0-20 \mathrm{~cm}$ e, em alguns estudos, tem se observado aumento dos estoques de COT em solo sob eucalipto, comparativamente às áreas nativas, quando as amostragens se estendem até $100 \mathrm{~cm}$ (Pulronik et al., 2009).

Similarmente ao COT, os estoques de C na FLL, nas camadas de $0-10$ e $10-20 \mathrm{~cm}$, foram maiores no solo sob Cerrado denso, seguido daquele obtido no solo sob Cerrado, em relação aos outros sistemas de uso do solo (Quadro 1). As reduções nos estoques de $\mathrm{C}$ da FLL observadas nos povoamentos de eucalipto, em relação ao Cerrado denso, variaram de $63 \%$ (PEUC-7) a $71 \%$ (PEUC-3) e de $72 \%$ a $81 \%$ (PEUC-3) nas camadas de $0-10$ e $10-20 \mathrm{~cm}$, respectivamente, e podem ser atribuídas ao preparo do solo realizado no plantio e na colheita da cultura, já que, anteriormente, a área se encontrava sob pastagem e, portanto, sem revolvimento. O revolvimento do solo causa a quebra dos agregados e expõe a matéria orgânica fisicamente protegida à atuação microbiana, aumentando as condições de aeração e, assim, contribuindo para a rápida decomposição, especialmente daquelas formas mais prontamente disponíveis ou lábeis, como o $\mathrm{C}$ da FLL (Tisdall \& Oades, 1982; Feller \& Beare, 1997; Lal et al., 2002; Mielniczuk et al., 2003; Leite et al., 2003; Bayer et al., 2006). Em relação ao cultivo de eucalipto, para diferentes idades, houve, de modo similar ao verificado para o COT, incremento nos estoques de $\mathrm{C}$ da FLL com o tempo de cultivo (PEUC$7>$ PEUC-6 > PEUC-3), nas camadas 0-10 e 10-20 cm (Quadro 1); isso é essencial, já que, embora sua proporção em relação a outros compartimentos de C, como as frações húmicas, seja menor, constitui-se em compartimento lábil, de rápida ciclagem e que pode favorecer a biota do solo (Lima et al., 2008; Laik et al., 2009). Considerando a camada de 0-20 cm, podese inferir que o estoque de C da FLL no eucalipto com

Quadro 1. Estoques de carbono orgânico total e de seus compartimentos em Latossolo Vermelho, nas camadas de 0-10, 10-20 e 0-20 cm, sob cultivo de eucalipto e fitofisionomias de Cerrado, em Brasília, DF

\begin{tabular}{|c|c|c|c|c|c|}
\hline Sistema de uso do solo & FLL ${ }^{(1)}$ & $\mathrm{FAF}^{(2)}$ & $\mathrm{FAH}^{(3)}$ & $\mathrm{FH}^{(4)}$ & $\operatorname{COT}^{(5)}$ \\
\hline & & & $0-10 \mathrm{~cm}$ & & \\
\hline Cerrado $^{(6)}$ & $2,76 \mathrm{~b}$ & $4,67 \mathrm{~b}$ & $5,25 \mathrm{~b}$ & $14,18 \mathrm{~b}$ & $25,85 \mathrm{~b}$ \\
\hline Cerrado denso & $3,25 \mathrm{a}$ & $5,87 \mathrm{a}$ & 6,41 a & $15,23 \mathrm{a}$ & 28,52 a \\
\hline Campo sujo & $1,46 \mathrm{c}$ & $1,98 \mathrm{c}$ & $2,89 \mathrm{c}$ & $11,75 \mathrm{~b}$ & $18,48 \mathrm{c}$ \\
\hline PEUC-3(7) & $0,94 \mathrm{~d}$ & $1,36 \mathrm{c}$ & $1,77 \mathrm{c}$ & $9,53 \mathrm{~b}$ & $15,19 \mathrm{e}$ \\
\hline PEUC-6 ${ }^{(8)}$ & $1,00 \mathrm{~d}$ & $1,43 \mathrm{c}$ & $1,83 \mathrm{c}$ & $11,44 \mathrm{~b}$ & $16,92 \mathrm{~d}$ \\
\hline \multirow[t]{2}{*}{ PEUC- $7^{(9)}$} & $1,20 \mathrm{c}$ & $1,63 \mathrm{c}$ & $2,03 \mathrm{c}$ & $13,03 \mathrm{~b}$ & $18,45 \mathrm{c}$ \\
\hline & & & $10-20 \mathrm{~cm}$ & & \\
\hline Cerrado & $1,49 \mathrm{~b}$ & $2,44 \mathrm{~b}$ & $2,68 \mathrm{~b}$ & $9,83 \mathrm{~b}$ & $18,87 \mathrm{~b}$ \\
\hline Cerrado denso & 2,83 a & 2,84 a & 3,68 a & 10,63 a & $19,87 \mathrm{a}$ \\
\hline Campo sujo & $0,60 \mathrm{~d}$ & $0,72 \mathrm{e}$ & $1,04 \mathrm{e}$ & $7,47 \mathrm{c}$ & $12,53 \mathrm{e}$ \\
\hline PEUC - 3 & $0,51 \mathrm{~d}$ & $1,04 \mathrm{~d}$ & $1,36 \mathrm{~d}$ & $6,84 \mathrm{~d}$ & $13,83 \mathrm{~d}$ \\
\hline PEUC - 6 & $0,58 \mathrm{~d}$ & $1,08 \mathrm{~d}$ & $1,43 \mathrm{~d}$ & $8,93 \mathrm{~b}$ & $14,04 \mathrm{~d}$ \\
\hline \multirow[t]{2}{*}{ PEUC - 7} & $0,77 \mathrm{c}$ & $1,41 \mathrm{c}$ & $1,62 \mathrm{c}$ & $9,76 \mathrm{~b}$ & $14,49 \mathrm{c}$ \\
\hline & & & $0-20 \mathrm{~cm}$ & & \\
\hline Cerrado & $4,25 \mathrm{~b}$ & $7,11 \mathrm{a}$ & $7,93 \mathrm{~b}$ & $24,01 \mathrm{~b}$ & $44,72 \mathrm{~b}$ \\
\hline Cerrado denso & $6,08 \mathrm{a}$ & $8,71 \mathrm{a}$ & $10,09 \mathrm{a}$ & $25,86 \mathrm{a}$ & $48,39 \mathrm{a}$ \\
\hline Campo sujo & $2,06 \mathrm{c}$ & $2,70 \mathrm{c}$ & $3,93 \mathrm{c}$ & $19,22 \mathrm{c}$ & $31,01 \mathrm{~cd}$ \\
\hline PEUC - 3 & $1,45 \mathrm{~d}$ & $2,40 \mathrm{~d}$ & $3,13 \mathrm{~d}$ & $16,37 \mathrm{~d}$ & $29,02 \mathrm{e}$ \\
\hline PEUC - 6 & $1,58 \mathrm{~d}$ & $2,51 \mathrm{~d}$ & $3,26 \mathrm{~d}$ & $20,37 \mathrm{c}$ & $30,96 \mathrm{~d}$ \\
\hline PEUC - 7 & $1,97 \mathrm{c}$ & $3,04 \mathrm{c}$ & $3,65 \mathrm{c}$ & $22,79 \mathrm{c}$ & $32,94 \mathrm{c}$ \\
\hline
\end{tabular}


sete anos (1,97 $\left.\mathrm{t} \mathrm{ha}^{-1}\right)$ provavelmente ainda aumentará, uma vez que estoques maiores dessa fração têm sido reportados em outros trabalhos, como o de Pulrolnik et al. (2009), em que se observaram, para um cultivo de 20 anos, $9,66 \mathrm{t} \mathrm{ha}^{-1}$.

Em termos de magnitude, as diminuições nos estoques de $\mathrm{C}$ da FLL, nas áreas com eucalipto, comparativamente às áreas nativas, foram mais expressivas do que as observadas no COT, realçando a maior sensibilidade desse compartimento às alterações no manejo. No entanto, essa maior sensibilidade foi influenciada pelo tipo de vegetação usada como referência (Cerrado, Cerrado denso e campo sujo) e pela idade do povoamento de eucalipto (três, seis e sete anos). Esses resultados podem ser corroborados por aqueles obtidos por Lima et al. (2008), que verificaram diferenças no estoque de $\mathrm{C}$ da FLL no solo sob mata nativa e povoamento de eucalipto aos seis anos de idade, na camada de $0-10 \mathrm{~cm}$, em Virginópolis, MG. Isso mostra que nem sempre a FLL deve ser usada isoladamente como indicador sensível das mudanças na qualidade do solo, em razão de diferentes usos e manejo, conforme também foi reportado por $\mathrm{Wu}$ et al. (2004), sendo importante, portanto, avaliar também as $\mathrm{SH}$ e o próprio COT, como estabelecido em outros estudos (Chen et al., 2004; Leifeld \& Kögel-Knabner, 2005; Lima et al., 2008). Resultados contraditórios podem ser decorrentes do fato de a FLL ser composta basicamente por resíduos vegetais parcialmente decompostos e, ainda, de essa fração ser fortemente influenciada pela quantidade e qualidade dos resíduos depositados sobre o solo (Six et al., 2002). Nesse contexto, Barreto et al. (2008) observaram que, com o aumento da idade do eucalipto, ocorre alteração na qualidade dos resíduos que são aportados ao solo, os quais se tornam mais recalcitrantes. Além dessas variações, há de se considerar que os estoques de $\mathrm{C}$ da FLL neste trabalho, tanto nas áreas nativas quanto naquelas sob cultivo de eucalipto, provavelmente tenham sido subestimados. Essa subestimação pode ter ocorrido em virtude do uso de solução de iodeto de sódio (NaI) como técnica de fracionamento densimétrico da matéria orgânica do solo, a qual apresenta menor eficiência de recuperação de C da FLL, se comparada à utilização de solução de politungstato de sódio, que promove a recuperação do C da FLL em estádios mais avançados de decomposição da MO e, portanto, é mais eficiente que a solução de $\mathrm{NaI}$, além de ter sido recomendada em estudo de fracionamento densimétrico da MO do solo (Conceição et al., 2007).

Os estoques de $\mathrm{C}$ das FAF, FAH e FH foram, conforme observado para o COT e FLL, maiores no solo sob Cerrado denso e Cerrado, comparativamente ao campo sujo e aos povoamentos de eucalipto, nas camadas de 0-10 e 10-20 cm (Quadro 1). As reduções nos estoques, considerando-se o Cerrado denso e os povoamentos de eucalipto, foram mais expressivas nas FAF: $72 \%$ (PEUC-7) a $76 \%$ (PEUC-3), na camada de $0-10 \mathrm{~cm}$, e $50 \%$ (PEUC-7) a $61 \%$ (PEUC-3), na camada de 10-20 cm); e FAH: $68 \%$ (PEUC-7) a $72 \%$ (PEUC-3), na camada de 0-10 cm, e $56 \%$ (PEUC-7) a $63 \%$ (PEUC-3), na camada de $10-20 \mathrm{~cm}$. Na camada de 0-10 cm, não houve diferenças nos estoques de $\mathrm{C}$ nas três frações húmicas, considerando-se o campo sujo e os povoamentos de eucalipto, diferentemente da camada de 10-20 cm, em que as três áreas cultivadas apresentaram maiores valores, sobretudo o PEUC-7 (1,41 t ha-1 (FAF); 1,62 t ha-1 (FAH); e $9,76 \mathrm{t} \mathrm{ha}^{-1}(\mathrm{FH})$, confirmando a mesma tendência verificada no COT e FLL.

Em relação à redução verificada no $\mathrm{C}$ da FLL, esses percentuais foram, em média, maiores na camada de 0-10 cm: $74 \%$ (FAF) e $70 \%(\mathrm{FAH})$; e menores na camada de $10-20 \mathrm{~cm}: 58 \%(\mathrm{FAH})$ e $60 \%(\mathrm{FAH})$. A $\mathrm{FH}$ foi pouco sensível às mudanças no manejo, com reduções nos estoques de $\mathrm{C}$ variando de 8 \% (PEUC-7, na camada de $10-20 \mathrm{~cm}$ ) a $37 \%$ (PEUC-3, na camada de 0-10 cm) (Quadro 1). Maiores estoques de $\mathrm{C}$ nas FAF e FAH em solo sob mata nativa, em comparação àqueles sob cultivo de eucalipto, nas profundidades de 0-5, 5-10 e 10-20 cm, também foram observados por Lima et al. (2008). Por outro lado, esses autores não constataram diferença no estoque de $\mathrm{C}$ da $\mathrm{FH}$ entre o solo sob mata nativa e sob eucalipto, nas profundidades de $0-5$ e $10-20 \mathrm{~cm}$, indicando, similarmente ao observado neste estudo, que as FAF e FAH são mais eficientes na detecção de mudanças de uso do solo quando comparadas à FH. Chen et al. (2004), em experimento conduzido na Austrália, verificaram que a substituição de mata nativa para o cultivo de pinheiro (Araucaria cunninghamii), aos 51 anos de idade, resultou na diminuição do estoque de $\mathrm{C}$ da FAF, enquanto o estoque de $\mathrm{C}$ da $\mathrm{FAH}$ ficou inalterado. Dessa forma, a FAF foi mais sensível para detectar mudança de uso do solo em relação à $\mathrm{FAH}$, o que pode ser decorrente do processo de polimerização de compostos húmicos. A FAH é mais polimerizada que a FAF, e essas duas frações, por sua vez, são menos polimerizadas que a FH (Stevenson, 1994). Nesse contexto, Six et al. (2002) salientam que compostos mais humificados apresentam maior complexidade bioquímica, devido à associação com as frações de argila do solo (estabilização coloidal), o que dificulta sua decomposição por microrganismos e favorece sua estabilização no solo, tal como ocorre com a FH, que está intimamente associada aos coloides inorgânicos do solo (Stevenson, 1994). Isso tem implicações na retenção de $\mathrm{C}$ no solo, visto que as frações mais humificadas são mais estáveis e apresentam maior tempo de residência no ambiente (Lima et al., 2008).

Em razão do emprego da análise dos componentes principais (Figura 1a,b), observou-se que o campo sujo e os povoamentos de eucalipto, aos três, seis e sete anos de idade, apresentaram comportamento similar quando considerado o conjunto das variáveis em estudo (compartimentos de C). Com base na ACP, evidenciouse que a substituição do Cerrado e Cerrado denso para o cultivo do eucalipto resultou em maiores decréscimos nos estoques de C orgânico do solo, sobretudo nos anos 

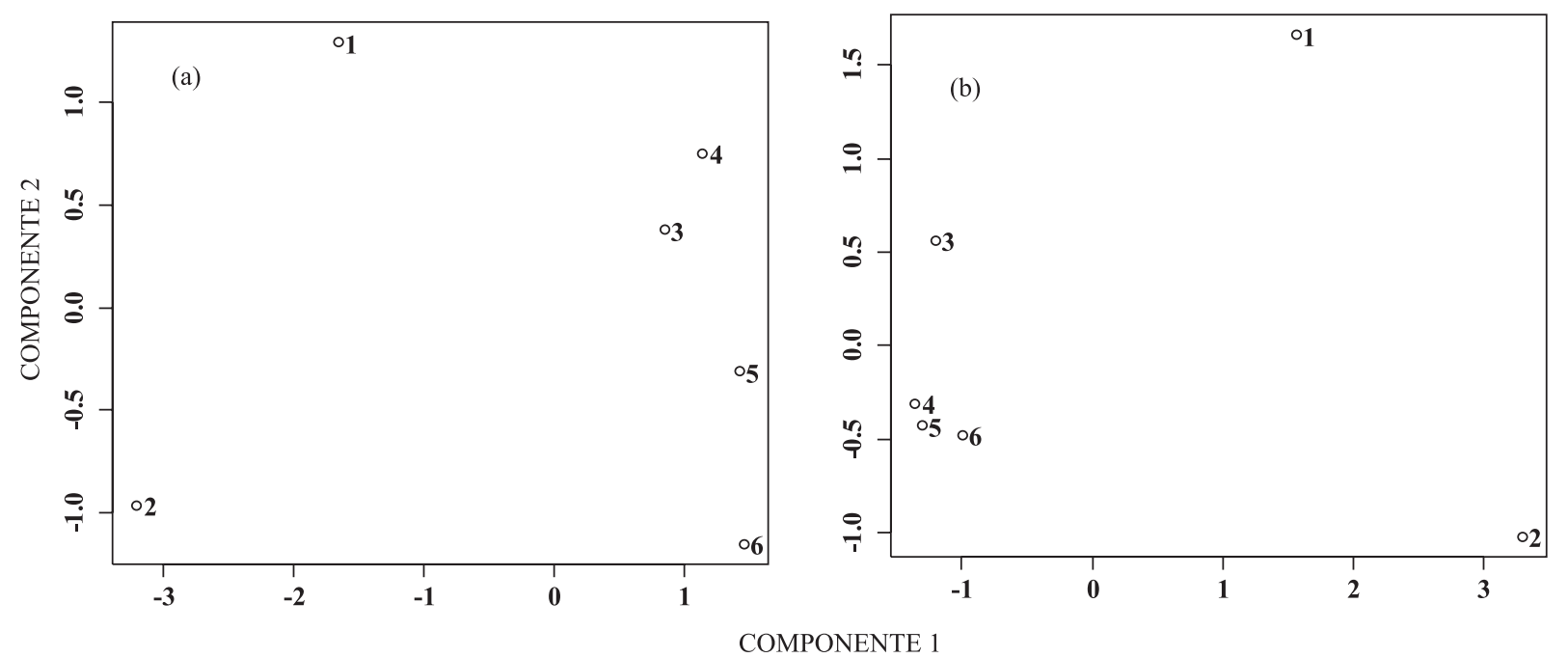

Figura 1. Dispersão dos diferentes sistemas de uso do solo e agrupamento dos dois primeiros componentes principais nas camadas de 0-10 cm (a) e 10-20 cm (b). 1: Cerrado; 2: Cerrado denso; 3: campo sujo; 4: povoamento de eucalipto aos três anos; 5: povoamento de eucalipto aos seis anos; 6: povoamento de eucalipto aos sete anos.

iniciais de implantação (PEUC-3), o qual apresentou menor similaridade, quando comparado com o sistema de uso do solo sob Cerrado e Cerrado denso. Por outro lado, o Cerrado e o Cerrado denso ficaram mais próximos entre si.

De acordo com Cruz \& Regazzi (1994), a distância euclidiana padronizada com valores próximos de zero significa maior similaridade entre os pares de valores avaliados. Nesse sentido, na camada de $0-10 \mathrm{~cm}$, verificou-se maior similaridade entre os seguintes sistemas de uso do solo: Cerrado e Cerrado denso; campo sujo e PEUC-3; PEUC-6 e campo sujo; e PEUC-
7 e PEUC-6, indicando que a substituição desse ecossistema para o cultivo do eucalipto pode resultar em menor impacto no conteúdo de $\mathrm{C}$ orgânico armazenado no solo. Na camada de 10-20 cm, observou-se maior similaridade entre Cerrado e Cerrado denso; campo sujo e PEUC-3; PEUC-3 e PEUC-6; e PEUC-7 e PEUC-3 (Quadro 2).

Constatou-se ainda que, com o aumento da idade do povoamento de eucalipto, diminuíram-se os valores das matrizes de dissimilaridade entre PEUC-7, Cerrado e Cerrado denso; os valores das matrizes em relação ao campo sujo aumentaram, sugerindo

Quadro 2. Matrizes de dissimilaridade da distância euclidiana padronizada, considerando-se as seis variáveis avaliadas em seis sistemas de uso do solo, nas camadas de 0-10 e 10-20 cm

\begin{tabular}{|c|c|c|c|c|c|c|}
\hline Sistema de uso do solo & Cerrado $^{(1)}$ & Cerrado denso & Campo Sujo & PEUC - $3^{(2)}$ & PEUC - $6^{(3)}$ & PEUC - $7^{(4)}$ \\
\hline \multicolumn{7}{|c|}{$0-10 \mathrm{~cm}$} \\
\hline Cerrado & 0,00 & 7,24 & 14,57 & 15,80 & 13,57 & 12,09 \\
\hline Cerrado denso & 7,24 & 0,00 & 20,39 & 21,79 & 19,05 & 16,83 \\
\hline Campo sujo & 14,57 & 20,39 & 0,00 & 1,69 & 3,01 & 6,36 \\
\hline PEUC - 3 & 15,80 & 21,79 & 1,69 & 0,00 & 4,18 & 7,68 \\
\hline PEUC - 6 & 13,57 & 19,05 & 3,01 & 4,18 & 0,00 & 3,51 \\
\hline PEUC - 7 & 12,09 & 16,83 & 6,36 & 7,68 & 3,51 & 0,00 \\
\hline \multicolumn{7}{|c|}{$10-20 \mathrm{~cm}$} \\
\hline Cerrado & 0,00 & 7,24 & 11,78 & 10,06 & 9,78 & 8,88 \\
\hline Cerrado denso & 7,24 & 0,00 & 17,52 & 15,24 & 14,86 & 13,81 \\
\hline Campo sujo & 11,78 & 17,52 & 0,00 & 3,06 & 3,51 & 4,43 \\
\hline PEUC - 3 & 10,06 & 15,24 & 3,06 & 0,00 & 0,47 & 1,54 \\
\hline PEUC - 6 & 9,78 & 14,86 & 3,51 & 0,47 & 0,00 & 1,11 \\
\hline PEUC - 7 & 8,88 & 13,81 & 4,43 & 0,54 & 1,11 & 0,00 \\
\hline
\end{tabular}

$\overline{{ }^{(1)} \text { Cerrado senso stricto. }}{ }^{(2)}$ PEUC -3: povoamento de eucalipto aos três anos de idade. ${ }^{(3)}$ PEUC-6: povoamento de eucalipto aos seis anos de idade. ${ }^{(4)}$ PEUC-7: povoamento de eucalipto aos sete anos de idade. 
recuperação no estoque de C no solo sob PEUC-7, em relação ao campo sujo. Pelo agrupamento de Tocher, utilizando a matriz de dissimilaridade do quadro 2, observou-se que, nas camadas de 0-10 e 10-20 cm, foram formados dois grupos (Quadro 3): grupo 1, com maior similaridade entre Cerrado e Cerrado denso; e grupo 2, com maior similaridade entre campo sujo e povoamentos de eucalipto de três, seis e sete anos.

Quadro 3. Agrupamento de Tocher, utilizando a medida euclidiana padronizada, em diferentes sistemas de uso do solo, nas camadas de 0-10 e $0-20 \mathrm{~cm}$

\begin{tabular}{ccc}
\hline Camada & Grupo & Uso do solo $^{(1)}$ \\
\hline \multirow{2}{*}{$0-10 \mathrm{~cm}$} & 1 & $1 ; 2$ \\
& 2 & $3 ; 4 ; 5 ; 6$ \\
$10-20 \mathrm{~cm}$ & 1 & $1 ; 2$ \\
& 2 & $3 ; 4 ; 5 ; 6$ \\
\hline
\end{tabular}

(1) 1: Cerrado; 2: Cerrado denso; 3: campo sujo; 4: povoamento de eucalipto aos três anos; 5 : povoamento de eucalipto aos seis anos; 6: povoamento de eucalipto aos sete anos.

\section{CONCLUSÕES}

1. As áreas sob Cerrado denso e Cerrado têm maiores estoques de $\mathrm{C}$ orgânico total e de $\mathrm{C}$ nos compartimentos da matéria orgânica do solo do que aquelas sob cultivo de eucalipto, nas camadas de 010 e 10-20 cm. Em ambas as camadas, há recuperação nos estoques de $\mathrm{C}$ do solo com o aumento do tempo de cultivo do eucalipto, indicando que, em médio e longo prazos, a biomassa aportada pela cultura poderá aumentar os níveis de matéria orgânica do solo.

2. O C da fração leve livre e das frações ácidos fúlvicos e ácidos húmicos foram mais sensíveis às alterações no manejo e podem ser adotados com indicadores de mudanças na dinâmica da matéria orgânica em função das práticas e dos sistemas de cultivo do solo adotados.

3. A análise multivariada, que considera todos os compartimentos conjuntamente, evidenciou maior similaridade entre o Cerrado denso e Cerrado, bem como entre o campo sujo, PEUC-3, PEUC-6 e PEUC7, nas camadas de $0-10$ e $10-20 \mathrm{~cm}$.

\section{LITERATURA CITADA}

BARRETO, P.A.B.; GAMA-RODRIGUES, E.F.; GAMARODRIGUES, A.C.; BARROS, N.F. \& FONSECA, S. Atividade microbiana, carbono e nitrogênio da biomassa microbiana em plantações de eucalipto, em seqüência de idades. R. Bras. Ci. Solo, 32:611-619, 2008.
BAYER, C.; MIELNICZUK, J. \& MARTIN-NETO, L. Efeito de sistemas de preparo e de cultura na dinâmica da matéria orgânica e na mitigação das emissões de $\mathrm{CO}_{2}$. R. Bras. Ci. Solo, 24:599-607, 2000.

BAYER, C.; LOVATO, T.; DIEKOW, J.; ZANATTA, J.A. \& MIELNICZUK, J. A method for estimating coefficients of soil organic matter dynamics based on long-term experiments. Soil Tillage Res., 91:217-226, 2006.

BOUILLET, J.; LACLAU, J.; ARNAUD, M.; M'BOU, A.T.; SAINT-ANDRÉ, L. \& JOURDAN, C. Changes with age in the spatial distribution of roots of Eucalyptus clone in Congo impact on water and nutrient uptake. For. Ecol. Manag., 171:43-57, 2002.

BUSTAMANTE, M.M.C.; CORBEels, M.; SCOPEL, E. \& ROSCOE, R. Soil carbon storage and sequestration potential in the Cerrado region of Brazil. In: LAL, R.; CERRI, C.C.; BERNOUX, M. \& CERRI, E. Carbon sequestration in soils of Latin America food products. New York, 2006. p.285-299.

CHEN, C.R.; XU, Z.H. \& MATHERSB, N.J. Soil carbon pools in adjacent natural and plantation forests of subtropical Australia. Soil Sci. Soc. Am. J., 68:282-291, 2004.

CONCEIÇÃO, P.C.; BOENI, M.; DIECKOW, J.; BAYER, C.; MARTIN-NETO, L. \& MIELNICZUK, J. Eficiência do politungstato de sódio no fracionamento densimétrico da matéria orgânica do solo. R. Bras. Ci. Solo, 31:1301-1310, 2007.

CRUZ, C.D. \& REGAZZI, A.J. Modelos biométricos aplicados ao melhoramento genético. Viçosa, MG, Universidade Federal de Viçosa, 1994. 394p.

R.DEVELOPMENT CORE TEAM. A language and environment for statistical computing. $\mathrm{R}$ foundation for Statical Computing. Vienna, 2008. Disponível em: <http:/ /R-project.org.2008>. Acesso em 19 nov. 2009.

EMPRESA BRASILEIRA DE PESQUISA AGROPECUÁRIA EMBRAPA. Centro Nacional de Pesquisa de Solos (Rio de Janeiro, RJ). Manual de métodos de análise de solo. 2.ed. Rio de Janeiro, 1997. 212 p.

EMPRESA BRASILEIRA DE PESQUISA AGROPECUÁRIA EMBRAPA. Centro Nacional de Pesquisa de Solos. Sistema brasileiro de classificação de solos. 2.ed. Rio de Janeiro, 2006. 306p.

FARIA, G.E.; BARROS, N.F.; NOVAIS, R.F.; SILVA, I.R. \& NEVES, J.C.L. Carbono orgânico total e frações da matéria orgânica do solo em diferentes distâncias do tronco de eucalipto. Sci. For., 36:265-277, 2008.

FELFILI, J.M.; SILVA-JUNIOR, M.C.; SEVILHA, A.C.; FAGG, C.W.; WALTER, B.M.T.; NOGUEIRA, P.E. \& REZENDE, A.V. Diversity, floristic and structural patterns of Cerrado vegetation in Central Brazil. Plant Ecol., 175:37-46, 2004.

FELLER, C. \& BEARE, M.H. Physical control of soil organic matter dynamics in the tropics. Geoderma, 79:69-116, 1997. 
FERREIRA, E.A.B.; RESCK, D.V.S.; GOMES, A.C. \& RAMOS, M.L.G. Dinâmica do carbono da biomassa microbiana em cinco épocas do ano em diferentes sistemas de manejo do solo no Cerrado. R. Bras. Ci. Solo, 31:1625-1635, 2007.

GUO, L.B. \& GIFFORD, R.M. Soil carbon stocks and use change: A meta analysis. Global Change Biol., 8:345-360, 2002 .

HAYNES, R.J. Labile organic matter fractions as central components of the quality of agricultural soils: An overview. Adv. Agron., 85:222-268, 2005.

LAL, R. The potential of soils of the tropics to sequester carbon and mitigate the greenhouse effect. Adv. Agron., 74:155192, 2002.

LAIK, R.; KUMAR, K.; DAS, D. \& CHATURVEDI, O.P. Labile soil organic matter pools in a calciorthent after 18 years of afforestation by different plantations Appl. Soil Ecol., 42:71-79, 2009

LEITE, L.F.C.; MENDONÇA, E.S. \& MACHADO, P.L.O.A Influence of organic and mineral fertilization on organic matter fractions of a Brazilian Acrisol under maize/ common bean intercrop. Austr. J. Soil Res., 45:25-32, 2007.

LEITE, L.F.C.; MENDONÇA, E.S.M.; MACHADO, P.L.O.A. \& MATOS, E.S. Total C and N storage and organic C pools of a Red-Yellow Podzolic under conventional and no tillage at the Atlantic Forest Zone, Southeastern Brazil. Austr. J. Soil Res., 41:717-730, 2003.

LEIFELD, J. \& KÖGEL-KNABNER, I. Soil organic matter fractions as early indicators for carbon stock changes under different land-use. Geoderma, 124:143-155, 2005.

LIANG, B.C.; McCONKEY, B.G.; SCHOENAU, J.; CURTIN, D.; CAMPBELL, C.A.; MOULLIN, A.P.; LAFOND, G.P. BRANDT, S.A. \& WANG, H. Effect of tillage and crop rotations onthe light fraction organic carbon mineralization in chernozemic soils of Sas-katchewan. Canadian J. Soil. Sci., 83:65-72, 2003

LIMA A.M.N.; SILVA, I.R.; NEVES, J.C.L.; NOVAIS, R.F.; BARROS, N.F.; MENDONÇA, E.S.; DEMOLINARI, M.S.M. \& LEITE, F.P. Frações da matéria orgânica do solo após três décadas de cultivo de eucalipto no Vale do Rio Doce-MG. R. Bras. Ci. Solo, 32:1053-1063, 2008.

MACHADO, P.L.O.A. Fracionamento físico do solo por densidade e granulometria para a quantificação de compartimentos da matéria orgânica do solo: Um procedimento para a estimativa pormenorizada do sequestro de carbono pelo solo. Rio de Janeiro, Embrapa Solos, 2002. 6p. (Comunicado Técnico, 9).

MIELNICZUK, J.; BAYER, C.; VEZZANI, F.M.; LOVATO, T.; FERNANDES, F.F. \& DEBARBA, L. Manejo de solo e culturas e sua relação com os estoques de carbono e nitrogênio do solo. In: CURI, N.; MARQUES, J.J.; GUILHERME, L.R.G.; LIMA, J.M.; LOPES, A.S. \& ALVAREZ V., V.H., eds. Tópicos em ciência do solo. Viçosa, MG, Sociedade Brasileira de Ciência do Solo, 2003. v.3. p.209-248.

MONTEIRO, M.T. \& GAMA-RODRIGUES, E.F. Carbono, nitrogênio e atividade da biomassa microbiana em diferentes estruturas de serapilheira de uma florestal natural. R. Bras. Ci. Solo, 28:819-826, 2004.
MOREIRA, A. \& MALAVOLTA, E. Dinâmica da matéria orgânica e da biomassa microbiana em solo submetido a diferentes sistemas de manejo na Amazônia Ocidental. Pesq. Agropec. Bras., 39:1103-1110, 2004.

PAUL, K.I.; POLGLASE, P.J.; NYAKUENGAMA, J.G. \& KHANNA, P.K. Change in soil carbon following afforestation. For. Ecol. Manag., 168:241-257, 2002.

PULROLNIK, K.; BARROS, N.F.; SILVA, I.R.; NOVAIS, R.F. \& BRANDANI, C.B. Estoques de carbono e nitrogênio em frações lábeis e estáveis da matéria orgânica de solos sob eucalipto, pastagem e Cerrado no vale do Jequitinhonha - MG. R. Bras. Ci. Solo, 33:1125-1136, 2009.

RANGEL, O.J.P. \& SILVA, C.A. Estoques de carbono e nitrogênio e frações orgânicas de Latossolo submetido a diferentes sistemas de uso e manejo. R. Bras. Ci. Solo, 31:1609-1623, 2007.

SILVA, F.A.S.E. \& AZEVEDO, C.A.V. A new version of the assistat-statistical assistance software. In: WORLD CONGRESS ON COMPUTERS IN AGRICULTURE, 4., Orlando, 2006. Anais... Orlando, American Society of Agricultural and Biological Engineers, 2006. p.393-396.

SIX, J.; CONANT, R.T.; PAUL, E.A. \& PAUSTIAN, K. Stabilization mechanisms of soil organic matter: Implications for C-saturation of soils. Plant Soil, 241:155176, 2002.

SOHI, S.P.; MAHIEU, N.; ARAH, J.R.M.; POWLSON, D.S.; MADARI, B. W. \& GAUNT, J.L. A procedure for isolating soil organic matter fractions suitable for modeling. Soil Sci. Soc. Am. J., 65:1121-1128, 2001.

STEVENSON, F.J. Humus chemistry: Genesis, composition, reactions. 2.ed. New York, J. Wiley \& Sons, 1994. 496p.

SWIFT, R.S. Method for extraction of IHSS soil fulvic and humic acids. In: SPARKS, D.L.; PAGE, A.L.; HELMKE, P.A.; LOEPPERT, R.H.; SOLTANPOUR, P.N.; TABATABAI, M.A.; JOHNSTON, C.T. \& SUMMER, M.E., eds. Methods of soil analysis. Chemical methods. Madison, Soil Science Society American Books, 1996. Part 3. p.10181020 .

TISDALL, J.M. \& OADES, J.M. Organic matter and waterstable aggregates in soils. J. Soil Sci., 33:141-163, 1982.

WU, T.; SCHOENAU, J.J.; LI, F.; QIAN, P.; MALHI, S.S.; SHI, Y. \& XU, F. Influence of cultivation and fertilization on total organic carbon and carbon fractions in soils from the Loess Plateau of China. Soil Tillage Res., 77:59-68, 2004.

YEOMANS, J.C. \& BREMNER, J.M. A rapid and precise method for routine determination of organic carbon in soil. Comm. Soil Sci. Plant Anal., 13:1467-1476, 1988.

ZAGAL, E.; MUNOZ C.; QUIROZ, M. \& CORDOVA, C. Sensitivity of early indicators for evaluating quality changes in soil organic matter. Geoderma, 151:191-198, 2009 .

ZINN, Y.L.; LAL, R. \& RESCK, D.V.S. Changes in soil organic carbon stocks under agriculture in Brazil. Soil Tillage Res., 84:28-40, 2005. 